\title{
Radar based rainfall nowcasting and its characteristic prediction based on spatially correlated random field, normalized duration line and Kalman filter algorithm
}

\author{
Ting $\mathrm{He}^{1, \text { a }}$, Chao Zhang ${ }^{1}$, Yi Zhang ${ }^{2}$ \\ ${ }^{1}$ Information Center, Ministry of Water Resources of P.R.China, 100053 Baiguang road 2 Lane 2, Beijing China \\ ${ }^{2}$ Beijing Jinshui Informtion Technology Development Co. Ltd, 100053 Baiguang road 2 Lane 2, Beijing China
}

\begin{abstract}
Rainfall is not only one of the most natural processes on the earth, but also an important factor of flood generation. Precise rainfall nowcasting can give an effective warning before hazards occur. This paper presented an ensemble nowcasting methodology which combined two deterministic nowcasting methods: PIV_Semi-Lagrangian and PIV_Lagrangian-Persistence and the spatial correlated random error field. For the deterministic nowcasting methods, the past velocity fields were estimated by Particle Image Velocimetry (PIV) method and the advection fields were extrapolated by Semi-Lagrange and Lagrange-Persistence schemes separately, then the forecasted errors at former time step were simulated by the spatially correlated random error field and were added to the next forecasting steps. Additionally, a predicting method for rain field property was proposed and a Kalman filter algorithm was also implemented for rain field's centre of mass prediction. The methodology was applied to 8 historical rainfall events occurred in North Rhine Westphalia (NRW), Germany by using high-resolution rainfall data acquired from C-band Essen radar belonging German Weather Service (DWD). Results showed that the promoted ensemble nowcasting methods and the rain field property predicting methods improved the forecasting accuracy obviously which confirmed their effectiveness.
\end{abstract}

\section{Introduction}

Precipitation processes a complex spatial and temporal structure on a wide range of scales [1]. It is well-known that rainfall nowcasting at high spatial and temporal resolution is one significant practice in short term flood forecast, especially in urban regions $[2,3]$. Therefore, understanding rainfall nowcasting uncertainties, making improvements in nowcasting systems is necessarily [4]. The now-going applied rainfall forecasting methodology is mostly depending on the high resolution image extrapolation way which takes the simple techniques in the extremes.

While the extrapolation techniques have been the mainstream of rainfall forecasting, uncertainties of forecasting give rise to the attentions of researchers as a result of chaotic nature of rainfall $([5,6,7])$. This study made an effort to subside the uncertainties in rainfall nowcasting by combining the extrapolating methods and spatially correlated random field. We also promoted a rainfall characteristics predicting methodology which is based on the Normalized duration line and Kalman filter methods for predicting the features of rain events.
Study area is the federal state of NRW (Figure 1). It is bordered by the German federal states of Lower Saxony to the north and north-east, Hessen to the east, and Rhineland-Palatinate to the south, and by the countries of Belgium to the south-west and the Netherlands to the west. NRW includes upland regions of North Eifel in the southern part, and mountains of the Sauerland in the south-east. The most important rivers that run at least partially through this region include: Rhine, Ruhr, Ems, Lippe and Weser. Two main types of landscapes can be found in NRW, namely the North German lowlands with elevations just a few meters above sea level, and the North German Low Mountain Range with elevations of up to $850 \mathrm{~m}$. The lowland areas comprise the Rhine-Ruhr area, which is one of the largest metropolitan areas with a population of approximating 10 million.

\section{Study area and data description}

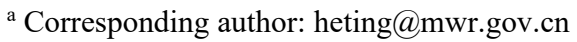




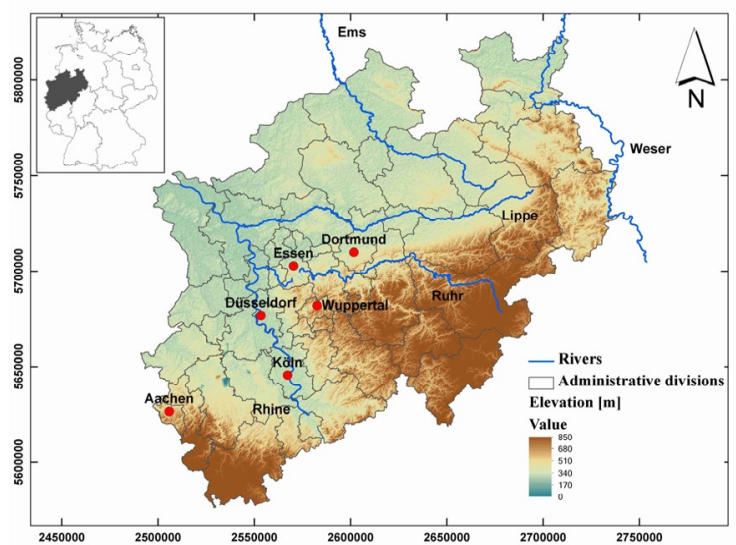

Figure 1. The state of North Rhine Westphalia, Germany

Data used in this study is produced from the C-band Essen radar attached to the radar network of DWD. The DWD radar network contains 16 dual-polarimetric Cband Doppler radar stations with a uniform scan strategy operated. They are distributed throughout Germany for complete coverage. They deliver radar volume scans (frequency: 800/1200 Hz, maximum range: $124 \mathrm{~km}$ ) every 15 minutes for the Doppler velocity and intensity volume scan (frequency: $500 \mathrm{~Hz}$, maximum range: 256 $\mathrm{km}$ ) and precipitation scan (frequency: $600 \mathrm{~Hz}$, maximum range: $150 \mathrm{~km}$ ) every 5 minutes for the precipitation echo, with a high spatial resolution of $1 \mathrm{~km}$ in range and $1^{\circ}$ in azimuth. For the selected data of this study, Essen radar operates a precipitation scan way with an elevation of $0.8^{\circ}$ and a range of $128 \mathrm{~km}$. Three rainy day's radar outputs (26 May 2007, 19 Jul 2008 and 26 Jul 2008) are picked with Plan Position Indicator (PPI) displaying type. As radar measures precipitation in an indirect manner up in the atmosphere, quality of radar data needs to be carefully considered. The methods for quality control of Essen radar data are presented by [8] for the following effects:

- Ground clutter and Speckle - For the correction of the ground clutter the following methods are used: a clutter map which is constituted from pixels where frequent clutter has been observed; a texture-based method to correct temporal clutter of high intensities observed; a reverse speckle filter to enhance pixels for which the value has been set to zero by the statistical clutter filter of the DWD and a spatial interpolation to calculate a value for each clutter pixel.

- Beam blockage - Essen radar suffers from partial beam blockage. As the affected area varies in time and space, for time intervals with a mainly homogeneous error the affected angles are corrected by matching $\mathrm{dBZ}$ values.

- Attenuation - The radar signal is attenuated conditioned by rain rate, but in extreme rainfall events the whole radar signal may be lost. Thus the detection of attenuation is much easier than its correction. By using stable ground clutter measurements, it is possible to correct attenuation with the "mountain return" method [9]. For this method an averaged clutter map is used as reference for the analysis and to determine the correction factor. If stable ground clutter areas are not available, it is possible to detect high $\mathrm{dBZ}$ values in the image. As correction algorithm for the attenuated areas behind these high dBZ values a cumulative gate-by-gate algorithm [10] can be used.

An open source package - Wradlib [11] is applied for projecting the raw radar image into a $256^{*} 256 \mathrm{~km}^{2}$ Cartesian map with $1 \mathrm{~km}$ resolution. The Z-R relationship presented as in equation (1) is used to transfer radar reflectivity $(Z)$ to rain rate $(R)$.

$$
Z=a R^{b}
$$

Two common Z-R relationships are used by German weather service in actual applications which are introduced by [12]: one is categorized for the RADOLAN product, and another one uses the constant $\mathrm{a}$ and $\mathrm{b}$ with values of 256 and 1.42. Though the DWD has stated that the categorized relationship statistically shows better results over long time periods, while the standard relationship can be more compatible to the local cases with a correction factor added [13]. Based on above considerations, we trust and apply the DWD's standard Z$\mathrm{R}$ relationship in radar reflectivity-rain rate conversion for the selected cases and the converted daily precipitation sums are presented as in Figure2.

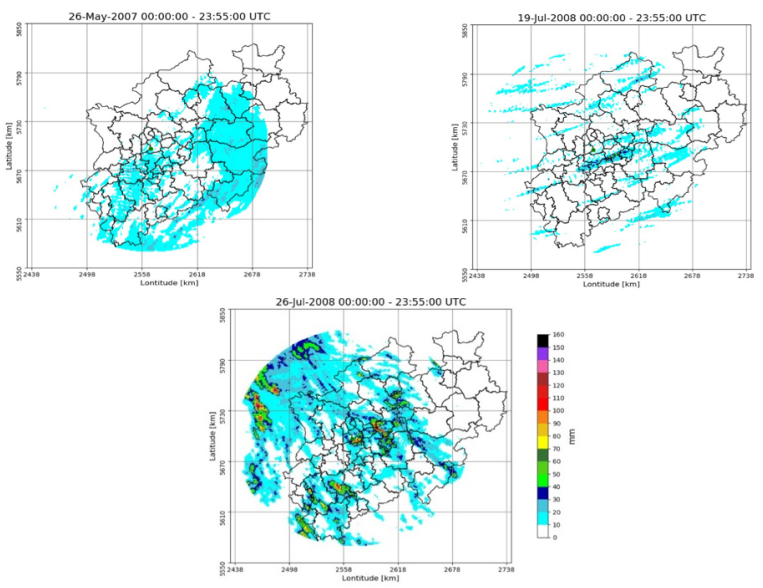

Figure 2. Precipitation sum of selected rainy days with 5 minutes accumulated data from Essen radar

\section{Methodology}

\subsection{Ensemble nowcasting method}

For rainfall nowcasting, if an error structure of forecasted rainfall is properly analysed with forecasting results at former time step and their error factors are found, then it would be useful to ongoing rainfall nowcasting with giving uncertainty. In this study, we presented this uncertainty by the spatially correlated random error field. The calculation procedure is listed as follows:

- Deducing the absolute nowcasting error field at former time step named as $E_{a}$ :

$$
E_{a}=R_{o b s}-R_{f c s t}
$$

where $R_{\text {obs }}$ is observed precipitation from radar image and $\mathrm{R}_{\mathrm{fcst}}$ is forecasted precipition. error vector $Y$ :

Generating the spatially correlated unit random

$$
Y=B w
$$

the matrix $B$ satisfies $K=B B$ when $K$ is symmetric and positive defined [14] and $\mathrm{w}$ is the uncorrelated random 
vector that obeys the standard Normal Distribution. Thus it can be found that $E\left[Y Y^{T}\right]=B I B^{T}=K$. The matrix $K$ is a covariance matrix obtained from the $E_{a}$ and it is decomposed into the symmetric matrix $B$ approximately by the Chebyshev polynomials.

- The error field at next time step can be shown as in equation (4)

$$
\left[\begin{array}{c}
E_{s, 1} \\
E_{s, 2} \\
E_{s, 3} \\
\vdots \\
E_{s, n}
\end{array}\right]=\left[\begin{array}{ccc}
s d_{0} & \cdots & 0 \\
\vdots & \ddots & \vdots \\
0 & \cdots & s d_{n}
\end{array}\right]\left[\begin{array}{c}
y_{1} \\
y_{2} \\
y_{3} \\
\vdots \\
y_{n}
\end{array}\right]+\left[\begin{array}{c}
m_{1} \\
m_{2} \\
m_{3} \\
\vdots \\
m_{n}
\end{array}\right]
$$

where, the $m_{i}$ and $s d_{i}$ are the mean and standard deviations of the current prediction error at former time step on grid $i$. The $y_{i}$ is the unit random error of the vector $Y$, and $E_{s, i}$ is the simulated error at the next time step.

- Then the nowcasting rainfall at grid $i$ at next time step can be presented as:

$$
R_{s, i}=R_{p, i}+E_{s, i}
$$

where $E_{s, i}$ is the simulated nowcasting error value on grid $i, R_{p, i}$ is the nowcasting rainfall from nowcasting methods.

\subsection{Normalized duration line}

During rainfall events, the rain field is generated and then grows with its size enlarged and rain rate increased. When the inner structures become saturated, the growth of rain field slows and keeps stable, then it is in the mature stage. At the end of the events, the size and rain rate of rain field decrease till it disappears and this is known as the dissipation stage.

Figure 3 presented a conceptual illustration of three stages of rain field over its life cycle where the vertical axis is the mean value of rain field's characteristics and the horizontal axis was the duration of field.

If we normalize all the rain field's trajectories, then a summary of stage variability of its properties following the normalized durations. For example, if the rain field's trajectories with different durations are extracted, then the 'Normalized Duration Lines' of their properties can be calculated where the range of normalized duration is set from 0 to 1 and we can give a heuristic summary that the growth stage of a rain field is between 0 and 0.4 , the mature stage is between 0.4 to 0.6 and the dissipation stage occurs between 0.6 to 1 .

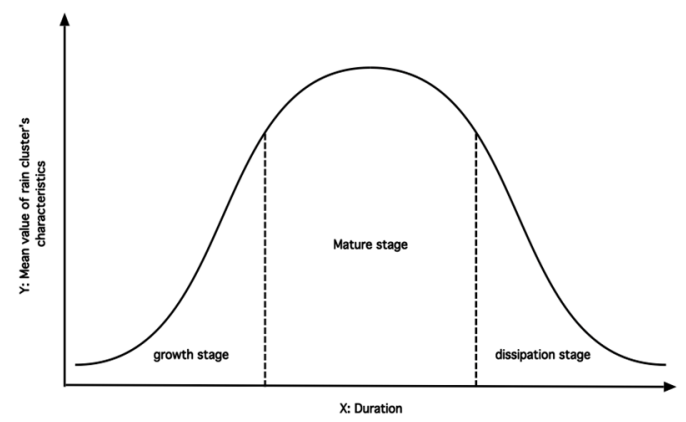

Figure 3. A conceptual illustration of three stages of rain cluster over its life cycle
Trajectories with multi durations can be normalized into one standard time axis by the 'Normalized duration lines' method and the temporal developments for rain cluster's characteristics can be presented. By applying regressive fitting method, the fitted curve can be calculated and presented as a parabola function, the coefficient parameters $a$ and $b$ of the parabola function can also be confirmed as in equation (6):

$$
V_{\text {properties }}=a t_{\text {normal }}^{2}+b t_{\text {normal }}
$$

where $V_{\text {properties }}$ is the normalized mean value of rain field's properties $a$ and $b$ are the coefficient parameters of the fitted parabola function, respectively. In the equation, $t_{\text {normal }}$ is the normalized duration of rain cluster. As in equation (6), predicting characteristics of rain cluster can be operated by equation (7):

$$
V_{f c s t}=
$$

$a V_{\text {now }}\left(t_{\text {normal }}+\frac{t_{f c s t}}{T}\right)\left(\left(m_{0.5}\left(t_{\text {norml }}\right)+\frac{t_{f c s t}}{T}\right)-\frac{b}{a}\right)$

where $V_{f c s t}$ is the predicted value at leading time $t_{f c s t}$, $m_{0.5}\left(t_{\text {norml }}\right)$ is the median value of the normalized duration for three stages which are presented in figure 3 (growth stage: 0.2 , mature stage: 0.5 and dissipation stage: $0.6)$. $V_{\text {now }}$ is the mean value of rain field's properties at previous time step. $T$ is the ratio of current time $t_{\text {now }}$ and median value of normalized duration. As for the practical application, the $V_{\text {now }}$ is averaged by the previous time step's property values before the forecast starts. At each forecasting step, a new $V_{\text {fcst }}$ is generated and is combined with the former time step's value for updating $V_{\text {now }}$, then the updated $V_{\text {now }}$ is inputted to the equation (7) and the new $V_{\text {fcst }}$ at next forecasting time is calculated. The rainfield's properties which the proposed predicting method applied are: 'area', 'cumulative rainfall', 'max rain rate per 5 minute' and 'mean rain rate per 5 minute'.

\subsection{Kalman filter for rain field centre of mass prediction}

The Kalman filter algorithm can be regarded as a statistical inversion method for solving linear filter of discrete data, in which an unknown state $x$ of the system is estimated combining with some noisy measurements [15].

When applying the Kalman filter algorithm to predict rain field center of mass, each rain field's position is treated as a discrete system. The unknown states $x$ can be regarded as an assembly of properties (e.g. center of mass, moving speed, area, cumulative rainfall). The goal of Kalman filter algorithm is to provide an optimal estimation for $x$ at the next time interval by the noise measurements at the previous time step and a dynamic process for the temporal development of state parameters. In the practical application, the measurements can be acquired from the outputs of precipitation objects identification and tracking algorithm. 


\section{Results and discussion}

Eight historical rain events occurred in NRW observed from Essen radar were selected for test the accuracy of ensemble nowcasting method as in table 1 .

As a comparsion, two PIV based nowcasting methods: PIV_Lagrangian-persistence and PIV_semi_Lagrangian, and two TREC based nowcasting methods: TREC_Lagrangian-persistence and TREC_semi_Lagrangian were also implemented [16].

Table 1. Selected historical rain events happened in NRW

\begin{tabular}{|c|c|c|c|}
\hline $\begin{array}{c}\text { Event } \\
\text { No. }\end{array}$ & Date & Time duration & $\begin{array}{c}\text { Maximum } \\
\text { intensity } \\
\text { [mm.5min-1] }\end{array}$ \\
\hline 1 & 26-May-2007 & $00: 00 \sim 02: 10$ & 22.1 \\
\hline 2 & 26-May-2007 & $19: 00 \sim 21: 10$ & 36 \\
\hline 3 & 19-Jul-2008 & $02: 00 \sim 04: 10$ & 7.7 \\
\hline 4 & 19-Jul-2008 & $13: 00 \sim 15: 10$ & 33.2 \\
\hline 5 & 19-Jul-2008 & $16: 00 \sim 18: 10$ & 53.9 \\
\hline 6 & 26-Jul-2008 & $00: 00 \sim 02: 10$ & 36 \\
\hline 7 & 26-Jul-2008 & $13: 00 \sim 15: 10$ & 49.7 \\
\hline 8 & 26-Jul-2008 & $16: 00 \sim 18: 10$ & 58.5 \\
\hline
\end{tabular}

Eight trajectories calculated from RCIT algorithm [16] were selected and eight ones for convective rain fields were selected for doing property prediction with a lead time up to one hour as in figure 4.

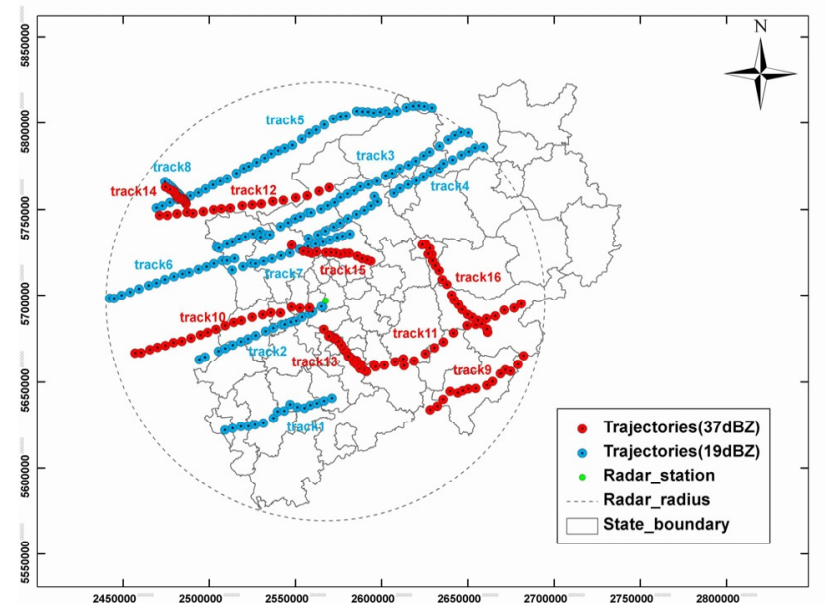

Figure 5. Selected trajectories which were generated from RCIT algorithm

\subsection{Verification of ensemble nowcasting methods}

We applied the SAL verification method [17] for quantifying the verification result of ensemble nowcasting method as in figure 6. It was showed that at low intensity nowcasting (threshold is $19 \mathrm{dBZ}$ ), median value of components $S$ and $A$ of two ensemble methods are more close to zero in comparison with SAL results of other nowcasting methods, which suggested that the structure and precipitation of forecasted objects from promoted ensemble nowcasting methods are more close to the observed ones and the forecasted object's center of mass from promoted methods were almost the same with other forecasting results.
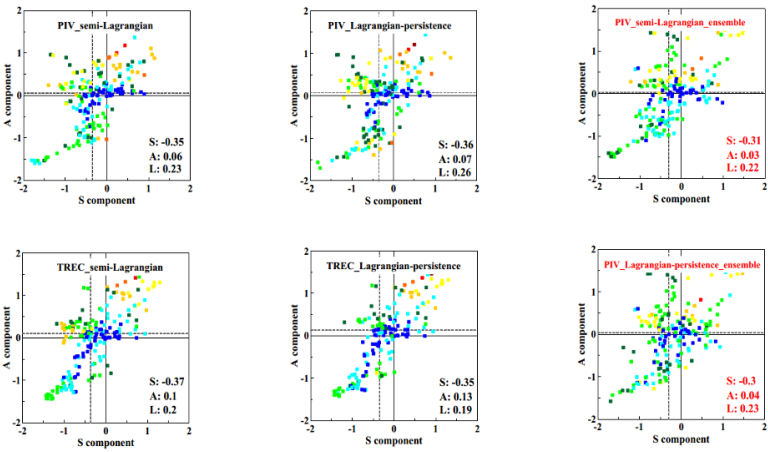

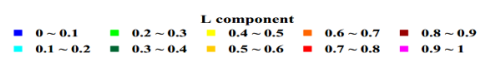

Figure 6. SAL verification results for all nowcasting methods at $19 \mathrm{dBZ}$, the dashed line at horizontal and vertical directions in the figure delegate the median value of $\mathrm{A}$ and $\mathrm{S}$ components, and the number in the figure is the median value of three SAL components

For high intensity nowcasting (threshold is $37 \mathrm{dBZ}$ ) as in figure 7, all three SAL components for promoted nowcasting methods were close to zero point compared to SAL results from reference nowcasting methods which meant that the forecasted precipitation objects from ensemble methods were more close to the observed ones, not only for their structure and precipitation, but also for their center of mass.
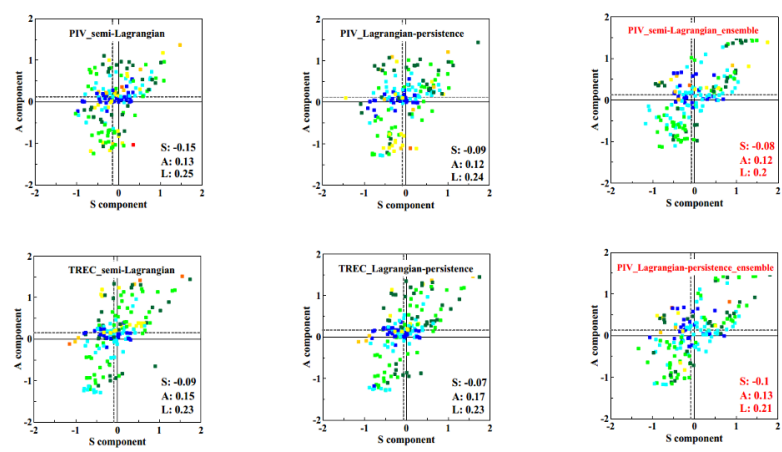

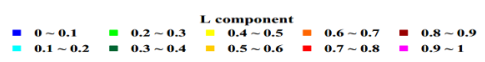

Figure 7. Same with figure 6 , but for nowcasting results at threshold of $37 \mathrm{dBZ}$

\subsection{Verification of rain field's property prediction results}

The normalized duration line and Kalman filter methods were applied to the selected trajectories for predicting the properties of rain field, the results were compared with classical extrapolating method. The root mean square error of prediction results were presented in table 2 .

Table 2. root mean square error of predicted results comparison for classical method (extrapolating method) and new method (normalized duration line and Kalman filter)

\begin{tabular}{|c|c|c|c|c|}
\hline \multirow{2}{*}{ Properties } & \multicolumn{2}{|c|}{$\begin{array}{c}\text { thresholds }> \\
\text { 19dBZ }\end{array}$} & \multicolumn{2}{c|}{$\begin{array}{c}\text { thresholds }> \\
\text { 37dBZ }\end{array}$} \\
\cline { 2 - 5 } & $\begin{array}{c}\text { classical } \\
\text { method }\end{array}$ & $\begin{array}{c}\text { new } \\
\text { method }\end{array}$ & $\begin{array}{c}\text { classical } \\
\text { method }\end{array}$ & $\begin{array}{c}\text { new } \\
\text { method }\end{array}$ \\
\hline Area $\left(\mathbf{k m}^{2}\right)$ & 126.3 & 55.8 & 91.9 & 56.4 \\
\hline $\begin{array}{c}\text { cum } \\
\text { precip(mm) }\end{array}$ & 120.7 & 45.6 & 214.6 & 151.6 \\
\hline
\end{tabular}




\begin{tabular}{|c|c|c|c|c|}
\hline $\begin{array}{c}\text { max } \\
\text { precip(mm) }\end{array}$ & 7.03 & 1.88 & 14.5 & 4.9 \\
\hline $\begin{array}{c}\text { mean } \\
\text { precip(mm/k } \\
\mathbf{m}^{2} \text { ) }\end{array}$ & 1.97 & 0.38 & 2.7 & 2.6 \\
\hline $\begin{array}{c}\text { Center of } \\
\text { masss_x(km) }\end{array}$ & 69.7 & 1.4 & 62.1 & 1.2 \\
\hline $\begin{array}{c}\text { Center of } \\
\text { mass_y(km) }\end{array}$ & 44.7 & 1.86 & 44.5 & 1.01 \\
\hline
\end{tabular}

It was showed that for low (threshold is $19 \mathrm{dBZ}$ ) and high (threshold is $37 \mathrm{dBZ}$ ) intensity prediction error from normalized duration line and Kalman filter were obviously less than results from extrapolating method, which proved that this two methods can be applied in actual nowcasting aspects.

\section{Conclusion}

In this study, we presented an ensemble nowcasting methodology which combined two deterministic nowcasting methods: PIV_Semi-Lagrangian and PIV Lagrangian-Persistence and the spatial correlated random error field. Additionally, a predicting method for rain field property was proposed and a Kalman filter algorithm was also implemented for rain field center of mass prediction. The application results showed that the promoted ensemble nowcasting methods and the rain field property predicting methods improved the forecasting accuracy obviously which confirmed their effectiveness. The future plan would be the more cases testing and actual applied in NRW.

\section{References}

1. Morin E., Yakir H., Hydrological impact and potential flooding of convective rain cells in a semiarid environment, Hydrological Sciences Journal, 59 (2014)

2. Collier, C.G., Flash flood forecasting: What are the limits of predictability? Quarterly Journal of the Royal Meteorological Society, 133(2007)

3. Schellart, A., Liguori, S., Kr“amer, S., Saul, A., RicoRamirez, M.A., Comparing quantitative precipitation forecast methods for prediction of sewer flows in a small urban area, Hydrological Sciences Journal, 59(2014)

4. Ebert, E., Wilson, L., Weigel, A., Mittermaier, M., Nurmi, P., Gill, P., G“ober, M., Joslyn, S., Brown, B., Fowler, T., Watkins, A., Progress and challenges in forecast verification, Meteorological Applications, 20(2013)

5. Kawamura A, Jinno K, Berndtsson R, Furukawa T., Real-time tracking of convective rainfall properties using a two-dimensional advection-diffusion model, Journal of Hydrology, 203(1997)

6. Georgakakos KP., Covariance propagation and updating in the context of real-time radar data assimilation by quantitative precipitation forecast models, Journal of Hydrology, 239(2000)
7. Grecu M, Krajewski WF., A large-sample investigation of statistical procedures for radar-based short-term quantitative precipitation forecasting, Journal of Hydrology, 239(2000)

8. Golz, C., Einfalt, T., Galli, G., Radar data quality control methods in Voltaire, Meteorologische Zeitschrift, 15(2006)

9. Sempere-Torres, D., Snchez-Diezma, R., Cordoba, M.A., Pascual, R., Zawadzki, I., An operational methodology to control radar measurements stability from mountain returns, in : 30th Conf. On Radar Met., Munich, Germany, 2001

10. Harrison,L, D., Driscoll, J, S., Improving precipitation estimates from weather radar using quality control and correction techniques, Meteorological Applications, 7 (2000)

11. Heistermann, M., Jacobi, S., Pfaff, T., Technical note: An open source library for processing weather radar data (wradlib), Hydrology and Earth System Sciences, 17(2013)

12. Weusthoff, T., Hauf, T., Basic characteristics of postfrontal shower precipitation rates, Meteorologische Zeitschrift, 17(2008)

13. Einfalt, T., Frerk, I., The challenge to estimate extreme precipitation for locations without rain gauges, in: Erad, (2012)

14. Davis MW., Generating large stochastic simulationThe matrix polynomial approximation method, Mathematical Geology 19, 2(1987)

15. Bar-Shalom, Y., Li, X. R., and Kirubarajan, T., Estimation with applications to tracking and navigation: theory algorithms and software, John Wiley \& Sons, (2004)

16. Ting He, Aizhong Hou, Thomas Einfalt, Property analysis of precipitation fields and feature based verification of short term rainfall forecasting using radar data for events in North Rhine Westphalia, Atmospheric Research, (2018) under review

17. Wernli, H., Paulat, M., Hagen, M., Frei, C., Sal-a novel quality measure for the verification of quantitative precipitation forecasts, Monthly Weather Review, 136(2008) 